\title{
ON NONDEGENERACY CONDITIONS FOR THE LEVI MAP IN HIGHER CODIMENSION: A SURVEY
}

\author{
LÉA BLANC-CENTI AND FRANCINE MEYLAN
}

\begin{abstract}
We compare various definitions of nondegeneracy of the Levi map for real submanifolds of higher codimension in $\mathbb{C}^{N}$ and discuss the generalization to higher codimension of the 2-jet determination for biholomorphisms in the hypersurface case proved by Chern and Moser in [10].
\end{abstract}

\section{INTRODUCTION}

Let $M$ be a real submanifold of $\mathbb{C}^{N}, p \in M$, and $\operatorname{Aut}(M, p)$ be the stability group of $M$ at point $p$, that is the set of (germs of) biholomorphisms $F$ fixing $p$ and such that $F(M) \subset M$.

For a real hypersurface in complex dimension 2, H. Poincaré initiated the study of the stability group by looking at Taylor series expansion: the condition $F(M) \subset M$ means that $\rho(F(z, w))_{\mid M}=0$, where $\rho$ is a defining function of $M$, and this equation gives some constraints on the Taylor series coefficients of $F$. The process was carried out much later in a significant manner by J.K. Moser for Levi non-degenerate hypersurfaces, to obtain the following 2-jet determination statement:

Theorem 1. 10] Let $M$ be a real-analytic hypersurface through a point $p$ in $\mathbb{C}^{N}$ with non-degenerate Levi form at $p$. Let $F, G$ be two germs of biholomorphic maps preserving $M$. Then, if $F$ and $G$ have the same 2-jets at $p$, they coincide.

The proof relies on the fact that the elements of $\operatorname{Aut}(M, p)$ have to satisfy an overdeterminated inhomogeneous system of partial differential equations, which is a very restrictive condition. But the result becomes false without any hypothesis on the Levi form, as one can see by considering the hyperplane $\operatorname{Im} w=0$ in $\mathbb{C}_{z}^{N} \times \mathbb{C}_{w}$, whose stability group at 0 is infinite dimensional.

Here, we are interested in 2- jet determination problems in higher codimension in the context of "nondegeneracy" of the Levi map. For $M$ being a submanifold of codimension $d$ in $\mathbb{C}^{N}$, the first step is to choose a convenient generalization of the Levi nondegeneracy condition. Various definitions appeared in the literature. Our aim is to survey these definitions, to compare them by giving many examples, and to explain why the definition introduced by Beloshapka in [4] seems the most relevant for us in the approach 
by model submanifolds. More precisely, we present a detailed explanation of the first part of his original proof in [4] which shows finite jet determination. Nevertheless, we explain why, following his proof, we can not conclude 2-jet determination in the general case. However we obtain 2-jet determination (using the same notion of nondegeneracy) in two special cases: in $\mathbb{C}^{4}$ without the assumption of smoothness through the technics of analytic discs (see [6]), and for codimension 2 in $\mathbb{C}^{N}$ through the technics developed in [18] (see [7]).

We point out that finite jet determination problems for submanifolds has attracted much attention. We refer in particular to the papers of Zaitsev [23], Baouendi, Ebenfelt and Rothschild [2], Baouendi, Mir and Rothschild [8], Ebenfelt, Lamel and Zaitsev [13, Lamel and Mir [19], Juhlin [15], Juhlin and Lamel [16], Mir and Zaitsev [20] in the real analytic case, Ebenfelt [11], Ebenfelt and Lamel [12, Kim and Zaitsev [17, Kolar, the second author and Zaitsev [18] in the $\mathcal{C}^{\infty}$ case, Bertrand and the first author [5], Bertrand, the first and the second authors [6] in the finitely smooth case.

The paper is organized as follows. In Section 2, we present the main protagonists and look at the model case. Section 3 is devoted to the various definitions of nondegeneracy. Finally, we give in Section 4 a detailed proof of the first part of the main theorem of [4] and discuss the 2-jet determination issue.

\section{Preliminaries}

2.1. General issue. Let $M \subseteq \mathbb{C}^{N}$ be a $\mathcal{C}^{3}$ real submanifold of (real) codimension $d \geq 1$ through $p$.

Some situations have to be excluded in order to get a finite dimensional stability group. For instance, the stability group of a complex submanifold of positive codimension is infinite dimensional: indeed, such a submanifold is given by $w=0$, and thus $(z, w) \mapsto(f(z), w)$ is a germ of automorphism at the origin as soon as $f(0)=0$ and $f$ is locally invertible at 0 . At the other extreme, $\operatorname{Aut}\left(\mathbb{R}^{N}, 0\right)$ is also infinite dimensional, because any mapping $F$ whose components are convergent power series with real coefficients, with $F(0)=0$ and the differential $d F_{0}$ is invertible, is a germ of automorphism.

To avoid these situations, we deal with a submanifold $M$ that is generic, and thus CR, assuming the CR bundle is of positive dimension. Under these hypotheses, up to a linear transformation, then using the implicit function theorem, we can assume that $p=0$ and the tangent space to $M \subseteq \mathbb{C}_{z}^{n} \times \mathbb{C}_{w}^{d}$ at 0 is $\operatorname{Im} w_{1}=\cdots=\operatorname{Im} w_{d}=0$, hence $M$ is given by

$$
\operatorname{Im} w=\left(\begin{array}{c}
\text { real quadratic terms in } \\
\operatorname{Re} w, \operatorname{Re} z, \operatorname{Im} z
\end{array}\right)+O\left(|(\operatorname{Re} w, z)|^{3}\right)
$$

Instead of grouping terms together according to their degree, we regroup them by weight (see [10]): a weight 2 is assigned to $w$ and a weight 1 is 
assigned to $z$. We get the $k$-th component of the second member of (11) decomposed in three parts:

- terms of weight 2: a real quadratic form in $z$, that is

$$
q_{k}(z)+\overline{q_{k}(z)}+(\text { Hermitian form in } z)
$$

with $q_{k}$ being a complex quadratic form;

- terms of weight at least 3: linear combination of $\left(\operatorname{Re} w_{i}\right)^{2},\left(\operatorname{Re} w_{i}\right)\left(\operatorname{Re} z_{j}\right)$ and $\left(\operatorname{Re} w_{i}\right)\left(\operatorname{Im} z_{j}\right)$ for $1 \leq i \leq d, 1 \leq j \leq n$;

- $O\left(|(\operatorname{Re} w, z)|^{3}\right)$, which would also consist of terms of weight at least 3 if the submanifold were real analytic.

After the local change of coordinates $z=z^{\prime}, w_{k}=w_{k}^{\prime}-2 i q_{k}(z)(1 \leq k \leq d)$, the only remaining terms of weight 2 are Hermitian, and $M$ is given locally by the following system of equations:

$$
\left\{\begin{array}{l}
\operatorname{Im} w_{1}={ }^{t} \bar{z} A_{1} z+\left(\begin{array}{c}
\text { linear combination in } \\
\left(\operatorname{Re} w_{i}\right)^{2}, z_{j} \operatorname{Re} w_{i}, \bar{z}_{j} \operatorname{Re} w_{i}
\end{array}\right)+O\left(|(z, \operatorname{Re} w)|^{3}\right) \\
\vdots \\
\operatorname{Im} w_{d}={ }^{t} \bar{z} A_{d} z+\left(\begin{array}{c}
\text { linear combination in } \\
\left(\operatorname{Re} w_{i}\right)^{2}, z_{j} \operatorname{Re} w_{i}, \bar{z}_{j} \operatorname{Re} w_{i}
\end{array}\right)+O\left(|(z, \operatorname{Re} w)|^{3}\right)
\end{array}\right.
$$

where $A_{1}, \ldots, A_{d}$ are Hermitian matrices of size $n$. Setting

$$
\left\langle z, z^{\prime}\right\rangle=\left({ }^{t} z A_{1} z^{\prime}, \ldots,{ }^{t} z A_{d} z^{\prime}\right)
$$

this system of equations can be written in a shortest way:

$$
\operatorname{Im} w=\langle\bar{z}, z\rangle+h(z, \operatorname{Re} w)
$$

where the Taylor expansion of $h$ at 0 consists of terms of weight at least 3 . We can also normalize the remaining term $h(z, \operatorname{Re} w)$ by asking every term of its Taylor expansion at 0 to have no pure terms, that is to involve both $z$ and $\bar{z}$ (see [1] and [9] section 7.2 for more details).

2.2. The Levi map. We recall that $A_{1}, \ldots, A_{d}$ are not uniquely determined but depend on the choice of the defining function of $M$. However, they contain some intrinsic geometric information on the submanifold because they are related to the Levi map, which is the generalization of the Levi form to the case of higher codimension.

For $p \in M$, let $T_{p} M$ be the tangent bundle to $M$ at $p, N_{p} M$ be the normal bundle to $M$ at $p$ (that is, the orthogonal complement of $T_{p} M$ in $T_{p}\left(\mathbb{R}^{2 N}\right)$, and denote by $\pi_{p}: T_{p}\left(\mathbb{R}^{2 N}\right) \rightarrow N_{p} M$ the orthogonal projection. Following [9], we then define the Levi map for all $p \in M$ :

Definition 2. The Levi map of $M$ at $p$ is $L_{p}: T_{p}^{(1,0)} M \rightarrow N_{p} M$ defined on the space of holomorphic vectors tangent to $M$ at $p$ by

$$
L_{p}\left(X_{p}\right)=\frac{1}{2 i} \pi_{p}(J[\bar{X}, X](p))
$$


where $X$ is any holomorphic vector field such that $X(p)=X_{p}$ and $J$ is the standard complex structure.

Note that the definition is valid for any $\mathrm{CR}$ submanifold of class $\mathcal{C}^{2}$, and that it is preserved under the action of CR-diffeomorphisms: let $M$, $M^{\prime}$ be CR submanifolds and $L^{M}, L^{M^{\prime}}$ their respective Levi maps, then if $F: M \rightarrow M^{\prime}$ is a CR-diffeomorphism we get

$$
\forall p \in M, \forall X_{p} \in T_{p}^{1,0} M, L_{p}^{M}\left(X_{p}\right)=d F_{p}^{-1}\left(L_{F(p)}^{M^{\prime}}\left(d F_{p} \cdot X_{p}\right)\right)
$$

Each component of $L_{p}$ being a Hermitian form, it is given by a unique sesquilinear form. We denote by $\mathcal{L}_{p}$ the corresponding sesquilinear map from $T_{p}^{(1,0)} M \times T_{p}^{(1,0)} M$ to $\mathbb{C} \otimes N_{p} M$, that is: $\forall X_{p}, Y_{p} \in T_{p}^{(1,0)} M$,

$$
\begin{aligned}
& 2 \mathcal{L}_{p}\left(X_{p}, Y_{p}\right)=\frac{1}{i} \pi_{p}(J[\bar{X}, Y](p)) \\
& =\left(L_{p}\left(X_{p}+Y_{p}\right)-L_{p}\left(X_{p}\right)-L_{p}\left(Y_{p}\right)\right) \\
& +i\left(L_{p}\left(X_{p}\right)+L_{p}\left(Y_{p}\right)-L_{p}\left(X_{p}+i Y_{p}\right)\right)
\end{aligned}
$$

where $\pi_{p}$ is extended between $\mathbb{C} \otimes T_{p}\left(\mathbb{R}^{2 N}\right)$ and $\mathbb{C} \otimes N_{p} M$.

In case $M$ is given by (2), we obtain a convenient expression in coordinates for the Levi map of $M$ at 0 ([9], section 10.2):

$$
\forall z \in \mathbb{C}^{n}, L_{0}(z)=\left({ }^{t} \bar{z} A_{1} z, \ldots,{ }^{t} \bar{z} A_{d} z\right)=\langle\bar{z}, z\rangle
$$

where $N_{0} M$ is identified with $\mathbb{R}^{d}$. Similarly, we get:

$$
\forall z, z^{\prime} \in \mathbb{C}^{n}, \mathcal{L}_{0}\left(z, z^{\prime}\right)=\left({ }^{t} \bar{z} A_{1} z^{\prime}, \ldots,{ }^{t} \bar{z} A_{d} z^{\prime}\right)=\left\langle\bar{z}, z^{\prime}\right\rangle
$$

2.3. The case of a quadric. To sort various conditions of non-degeneracy, we begin with the simplest case of a quadric submanifold $Q$, for which there is only a Hermitian part in $z$ :

$$
\left\{\begin{array}{c}
\operatorname{Im} w_{1}={ }^{t} \bar{z} A_{1} z \\
\vdots \\
\operatorname{Im} w_{d}={ }^{t} \bar{z} A_{d} z
\end{array}\right.
$$

It is easy to see that we need to put at least two conditions on the $A_{j}$ if we expect $\operatorname{Aut}(Q, 0)$ to be finite dimensional. This will give a motivation for the nondegeneracy condition in the sense of Beloshapka.

Lemma 3. If $\operatorname{Aut}(Q, 0)$ is finite dimensional, then

(a) the Hermitian matrices $A_{1}, \ldots, A_{d}$ are linearly independent (equivalently on $\mathbb{R}$ or $\mathbb{C}$ )

(b) $\bigcap_{j=1}^{d} \operatorname{Ker} A_{j}=\{0\}$ 


\section{Remark 4.}

- Condition $(\mathfrak{a})$ cannot be satisfied for $d>n^{2}$, the dimension of the space of Hermitian matrices of size $n$.

- Condition $(\mathfrak{b})$ is equivalent to say that if $\left\langle z, z^{\prime}\right\rangle=0$ for all $z^{\prime}$, then we get $z=0$.

Proof. Assume first that $(\mathfrak{a})$ is not satisfied, that is for instance $\exists \lambda_{1}, \ldots, \lambda_{d-1} \in$ $\mathbb{R}$ such that $A_{d}=\sum_{i=1}^{d-1} \lambda_{i} A_{i}$. After the complex linear change of coordinates

$$
\left(z_{1}, \ldots, z_{n}, w_{1}, \ldots, w_{d}\right) \mapsto\left(z_{1}, \ldots, z_{n}, w_{1}, \ldots, w_{d-1}, w_{d}-\sum_{i=1}^{d-1} \lambda_{i} w_{i}\right)
$$

equation (5) becomes

$$
\left\{\begin{array}{l}
\operatorname{Im} w_{1}={ }^{t} \bar{z} A_{1} z \\
\quad \vdots \\
\operatorname{Im} w_{d-1}={ }^{t} \bar{z} A_{d-1} z \\
\operatorname{Im} w_{d}=0
\end{array}\right.
$$

The stability group at 0 thus contains the following mappings

$$
\left(z_{1}, \ldots, w_{d}\right) \mapsto\left(z_{1}, \ldots, w_{d-1}, f\left(w_{d}\right)\right)
$$

for $f\left(w_{d}\right)=\sum_{k=1}^{+\infty} a_{k} w_{d}^{k}$ being any convergent power serie with $a_{1} \neq 0$ and $a_{k} \in \mathbb{R}$ for all $k$.

Assume now that $(\mathfrak{b})$ is not satisfied: there exists some $z$ of norm 1 in $\bigcap_{j=1}^{d} \operatorname{Ker} A_{j}$. We can always assume $z=(1,0, \ldots, 0)$ up to a unitary transformation, that is, the first column of every $A_{j}$ is zero, and so is the first raw. This means that $\langle z, z\rangle=\left({ }^{t} \bar{z} A_{1} z, \ldots,{ }^{t} \bar{z} A_{d} z\right)$ does not depend on $z_{1}$, hence the stability group at 0 contains mappings as

$$
\left(z_{1}, \ldots, w_{d}\right) \mapsto\left(f\left(z_{1}\right), z_{2}, \ldots, z_{n}, w_{1}, \ldots, w_{d}\right)
$$

where $f$ is any holomorphic function in a neighborhood of 0 such that $f(0)=$ 0 and $f^{\prime}(0) \neq 0$.

\section{Non-Degeneracy in the Sense of BeloshapkA}

Lemma 3 leads to the following.

\subsection{The definition and its properties.}

Definition 5. The generic $\mathcal{C}^{3}$ real submanifold $M$ of $\mathbb{C}^{N}$ given by (2) is said Levi non-degenerate at 0 (in the sense of Beloshapka) if conditions (a) and $(\mathfrak{b})$ are both satisfied. 
This convenient definition was introduced in [3, 4]. We will check later that, even if it seems to depend on the defining function of $M$, the Levi non-degeneracy is actually a biholomorphic invariant. We first investigate the relations between $(\mathfrak{a})$ and $(\mathfrak{b})$.

In the hypersurface case $d=1,(\mathfrak{a})$ means that the only matrix $A_{1}$ is non zero, and $(\mathfrak{b})$ means it is invertible; thus $(\mathfrak{b})$ is exactly the usual Levi nondegeneracy condition for hypersurfaces and it obviously implies $(\mathfrak{a})$. Note that $(\mathfrak{a})$ and $(\mathfrak{b})$ are equivalent only for a real hypersurface in $\mathbb{C}^{2}(n=d=1)$.

Lemma 6. In codimension $d \geq 2$,

- (b) never implies $(\mathfrak{a})$.

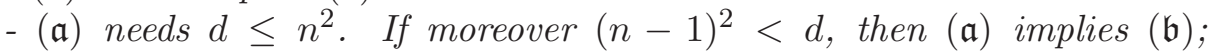
otherwise the implication is always false.

Proof. The first point is obvious, since one can choose $A_{1}$ invertible and $A_{2}=\cdots=A_{d}=0$. The beginning of the second point comes from Remark 4. If $(\mathfrak{a})$ is satisfied and $(\mathfrak{b})$ is not satisfied: then, up to a change of basis, the first raw and the first column of the $A_{i}$ are zero, and the dimension $d$ of the vector space generated by the $A_{i}$ is thus less than the dimension of Hermitian matrices of size $(n-1)$, that is, $(n-1)^{2}$.

If $d \leq(n-1)^{2}$ : then $n \geq 2$, and we can choose $d$ linear independent Hermitian matrices $B_{1}, \ldots, B_{d}$ of size $n-1$. Set $A_{i}=\left(\begin{array}{ccc}B_{k} & & 0 \\ 0 & \ldots & 0\end{array}\right)$ : the $A_{i}$ are linearly independent but $(\mathfrak{b})$ is not satisfied.

Thus, what are the first cases to explore for $(\mathfrak{a})$ and $(\mathfrak{b})$ being satisfied? In $\mathbb{C}^{2}$ resp. $\mathbb{C}^{3}$, it remains only the hypersurface case $(n=d=1$ or $n=2, d=$ $1)$; in $\mathbb{C}^{4}$, the hypersurface case $(n=3, d=1)$ or $n=d=2$. So the first interesting situation for higher codimension concerns the real codimension 2 in $\mathbb{C}^{4}$. We observe that in this case we just have to check $(\mathfrak{a})$, since $(\mathfrak{a})$ implies $(\mathfrak{b})$ (because $\left.d>(n-1)^{2}\right)$.

3.2. Other non-degeneracy conditions. Among the wide literature concerning finite jet determination problems, various (non equivalent) generalizations in higher codimension of the notion of non-degeneracy appear:

- non-degeneracy in [23, 1] is exactly condition $(\mathfrak{b})$;

- Levi non-degeneracy in the sense of Tumanov [22] means, using (4), that there exists a (real) linear combination $\sum \lambda_{j} A_{j}$ that is invertible; or equivalently, that the conormal bundle $N^{*} M$ is totally real at any point $\left(0, \sum \lambda_{j} \partial \rho_{j}(0)\right)$ where $\left(\rho_{j}\right)$ is a set of defining functions for $M$; this obviously implies $(\mathfrak{b})$ (but not $(\mathfrak{a})$, except in the hypersurface case, since one can choose $A_{1}$ invertible and $\left.A_{2}=\ldots=A_{d}=0\right)$.

Note that the Levi non-degeneracy in the sense of Beloshapka does not give the Levi non-degeneracy in the sense of Tumanov: 
Example 7. 4] In $\mathbb{C}^{6}$, consider the following codimension 3 real submanifold:

$$
\left\{\begin{array}{l}
\operatorname{Im} w_{1}=\left|z_{1}\right|^{2} \\
\operatorname{Im} w_{2}=2 \operatorname{Re}\left(z_{1} \bar{z}_{2}\right) \\
\operatorname{Im} w_{3}=2 \operatorname{Re}\left(z_{1} \bar{z}_{3}\right)
\end{array}\right.
$$

that is

$$
A_{1}=\left(\begin{array}{ccc}
1 & 0 & 0 \\
0 & 0 & 0 \\
0 & 0 & 0
\end{array}\right) \quad A_{2}=\left(\begin{array}{ccc}
0 & 1 & 0 \\
1 & 0 & 0 \\
0 & 0 & 0
\end{array}\right) \quad A_{3}=\left(\begin{array}{ccc}
0 & 0 & 1 \\
0 & 0 & 0 \\
1 & 0 & 0
\end{array}\right)
$$

Conditions $(\mathfrak{a})$ and $(\mathfrak{b})$ are satisfied, but no linear combination of $A_{1}, A_{2}, A_{3}$ is invertible.

3.3. Geometric characterizations. Conditions $(\mathfrak{a})$ and $(\mathfrak{b})$ have been defined in terms of a reduced equation of $M$. But they are equivalent to some geometric conditions, proving actually they are biholomorphically invariant. More precisely:

Proposition 8. The following statements are equivalent to condition $(\mathfrak{a})$ :

i) the image of $L_{p}: T_{p}^{1,0} M \rightarrow N_{p} M$ is not included in a hyperplan;

ii) the Levi cone of $M$ at $p$ (that is, the linear hull of the image of $L_{p}$ in $\left.N_{p} M\right)$ has a non empty interior;

iii) $M$ is of finite type at $p$ with 2 the only Hörmander number.

Remark 9. According to (3), the first point implies (a) is biholomorphically invariant. The second point means $M$ has a generating Levi form at $p$ in the sense of [22].

Proof.

i) Expressing the Levi map in convenient coordinates (44),

$$
L_{p}\left(T_{p}^{(1,0)} M\right)=\left\{\left({ }^{t} \bar{z} A_{1} z, \ldots,{ }^{t} \bar{z} A_{d} z\right) \mid z \in \mathbb{C}^{n}\right\} \subset \mathbb{R}^{d}
$$

is included in a hyperplane if and only if there exists $\left(\lambda_{1}, \ldots, \lambda_{d}\right) \in$ $\mathbb{R}^{d} \backslash\{(0, \ldots, 0)\}$ such that

$$
\forall z \in \mathbb{C}^{n}, \lambda_{1}{ }^{t} \bar{z} A_{1} z+\ldots+\lambda_{d}{ }^{t} \bar{z} A_{d} z=0
$$

i.e. the Hermitian matrix $\lambda_{1} A_{1}+\ldots+\lambda_{d} A_{d}$ is zero.

ii) Let $\Gamma_{p}$ be the Levi cone of $M$ at $p$ : by definition, $\Gamma_{p}$ is included in a hyperplane if and only if $L_{p}\left(T_{p}^{(1,0)} M\right)$ is included in a hyperplane. But since $\Gamma_{p}$ is convex, it is included in a hyperplane if and only if its interior is empty.

iii) Recall that $M$ being of finite type at $p$ with 2 the only Hörmander number means that there exist $\mathrm{CR}$ vector fields $L_{1}, \ldots, L_{j}$ such that $L_{1}, \ldots, L_{j}, \overline{L_{1}}, \ldots, \overline{L_{j}}$ and their Lie brackets generate the complexified tangent space $\mathbb{C} \otimes T_{p} M$. Or, equivalently, that there exists a basis of vector fields of $T^{(1,0)} M: X_{1}, \ldots, X_{n}$, such that $X_{1}(p), \ldots, X_{n}(p)$, 
$\overline{X_{1}(p)}, \ldots, \overline{X_{n}(p)}$ and the $\left[\overline{X_{i}}, X_{j}\right](p)$ generate $\mathbb{C} \otimes T_{p} M$.

By definition of the sesquilinear Levi map, this happens if and only if $\operatorname{Im}\left(\mathcal{L}_{p}\right):=\left\{\mathcal{L}_{p}\left(X_{p}, Y_{p}\right) \mid X_{p}, Y_{p} \in T_{p}^{(1,0)} M\right\}$ generates $\mathbb{C} \otimes N_{p} M$. Since $\mathcal{L}_{p}\left(X_{p}, Y_{p}\right)$ can be written as a complex linear combination of values of $L_{p}$, we get $\operatorname{Im}\left(L_{p}\right) \subset \operatorname{Im}\left(\mathcal{L}_{p}\right) \subset \operatorname{Span}_{\mathbb{C}}\left(\operatorname{Im}\left(L_{p}\right)\right)$ and thus $\operatorname{Span}_{\mathbb{C}}\left(\operatorname{Im}\left(\mathcal{L}_{p}\right)\right)=\operatorname{Span}_{\mathbb{C}}\left(\operatorname{Im}\left(L_{p}\right)\right)=\mathbb{C} \otimes \operatorname{Span}_{\mathbb{R}}\left(\operatorname{Im}\left(L_{p}\right)\right)$. Finally, $\operatorname{Span}_{\mathbb{C}}\left(\operatorname{Im}\left(\mathcal{L}_{p}\right)\right)=\mathbb{C} \otimes N_{p} M$ if and only if $\operatorname{Span}_{\mathbb{R}}\left(\operatorname{Im}\left(L_{p}\right)\right)=N_{p} M$.

Remark 10. If the sesquilinear Levi map $\mathcal{L}_{p}$ of $M$ is surjective, then the first condition holds. But in contrary of what is said in Lemma 4.3.26 page 108 of [1], the surjectivity of the sesquilinear Levi map $\mathcal{L}_{p}$ is not equivalent to $(\mathfrak{a})$, as we will show in the following example.

Example 11. Take in $\mathbb{C}^{6}$,

$$
\left\{\begin{array}{l}
\operatorname{Im} w_{1}=\left|z_{1}\right|^{2} \\
\operatorname{Im} w_{2}=\left|z_{2}\right|^{2} \\
\operatorname{Im} w_{3}=2 \operatorname{Re}\left(z_{1} \bar{z}_{2}\right) \\
\operatorname{Im} w_{4}=2 \operatorname{Im}\left(z_{1} \bar{z}_{2}\right)
\end{array}\right.
$$

Indeed,

$$
A_{1}=\left(\begin{array}{ll}
1 & 0 \\
0 & 0
\end{array}\right), \quad A_{2}=\left(\begin{array}{ll}
0 & 0 \\
0 & 1
\end{array}\right), \quad A_{3}=\left(\begin{array}{ll}
0 & 1 \\
1 & 0
\end{array}\right), \quad A_{4}=\left(\begin{array}{cc}
0 & i \\
-i & 0
\end{array}\right)
$$

They are linearly independent but $\mathcal{L}_{0}: \mathbb{C}^{2} \times \mathbb{C}^{2} \rightarrow \mathbb{C}^{4}$ is expressed by

$$
\mathcal{L}_{0}\left(z, z^{\prime}\right)=\left(\bar{z}_{1} z_{1}^{\prime}, \bar{z}_{2} z_{2}^{\prime}, \bar{z}_{1} z_{2}^{\prime}+\bar{z}_{2} z_{1}^{\prime}, i \bar{z}_{1} z_{2}^{\prime}-i \bar{z}_{2} z_{1}^{\prime}\right)
$$

and after a composition with a linear invertible map, we obtain

$$
\left(z, z^{\prime}\right) \mapsto\left(\bar{z}_{1} z_{1}^{\prime}, \bar{z}_{2} z_{2}^{\prime}, \bar{z}_{1} z_{2}^{\prime}, \bar{z}_{2} z_{1}^{\prime}\right)
$$

This map is not surjective since it takes its values in

$$
\left\{\left(t_{1}, t_{2}, t_{3}, t_{4}\right) \in \mathbb{C}^{4} \mid t_{1} t_{2}=t_{3} t_{4}\right\}
$$

Remark 12. Note that the surjectivity of $\mathcal{L}_{p}$ does not imply the surjectivity of $L_{p}$, even if $(\mathfrak{a})$ and $(\mathfrak{b})$ are satisfied: for instance, with $d=n=2$,

$$
A_{1}=\left(\begin{array}{ll}
1 & 0 \\
0 & 0
\end{array}\right) \quad \text { et } \quad A_{2}=\left(\begin{array}{ll}
0 & 0 \\
0 & 1
\end{array}\right)
$$

The image of $\mathcal{L}_{0}$ is $\mathbb{C}^{2}$ but the image of $L_{0}$ is $\left(\mathbb{R}^{+}\right)^{2}$.

Remark 13. Condition $(\mathfrak{b})$ is biholomorphically invariant since it means that

$$
\left(\forall X \in T_{p}^{(1,0)} M, \mathcal{L}_{p}(Y, X)=0\right) \quad \Longrightarrow \quad Y=0
$$

We recall the following definition. 
Definition 14. 1] A smooth generic submanifold $M \subset \mathbb{C}^{N}$ is holomorphically nondegenerate (in the formal sense) at $p \in M$ if there is no nontrivial formal holomorphic vector field at $p$ tangent to $M$.

Remark 15. Note that if $M$ is real analytic and holomorphically nondegenerate (in the formal sense) at $p$, then $M$ is holomorphically nondegenerate at $p$ in the classical sense, that is, there is no nontrivial holomorphic vector field at $p$ tangent to $M$. See Proposition 11.7.4 in [1].

The following proposition shows that condition $(\mathfrak{b})$ has also some geometric meaning.

Proposition 16. Condition $(\mathfrak{b})$ implies that $M$ is holomorphically non degenerate (in the formal sense), provided that $M$ is smooth.

Proof. Using Proposition 2.1 in [23] and Theorem 11.5.1 in [1], we deduce that $Q$ given by (5) is holomorphically nondegenerate, and hence $M$ is holomorphically nondegenerate.

\section{Discussing the Theorem of Beloshapka}

Let $M$ be a generic smooth real submanifold in $\mathbb{C}^{N}$. The following statement obtained in 1989 by Beloshapka shows that the non-degeneracy condition in the sense of Beloshapka was the good hypothesis for finite-jet determination for $M$ of finite type with 2 the only Hörmander number. Later on, in 1997, Zaitsev obtained the bound $2(1+\operatorname{codim} M)$ for the number of derivatives needed in the case where $M$ is real-analytic. (See Theorem 1.2 in [23]).

Theorem 17. 4

Let $M$ be a generic smooth real submanifold in $\mathbb{C}^{N}$, and $q \in M$. Assume $M$ is nondegenerate in the sense of Beloshapka at $q$ : then there exists some $k$, only depending on the Levi map of $M$ at point $q$, such that the elements of $\operatorname{Aut}(M, q)$ are uniquely determined by the values of the derivatives up to order $k$ at point $q$.

Note that the result was stated for real analytic submanifolds, but the proof given in [4] actually works for smooth $\left(\mathcal{C}^{\infty}\right)$ submanifolds, but does not give a precise estimate of $k$. In this section, we fulfill the details of this original proof. As before, we denote by $d$ the real codimension of $M$, described by (2):

$$
v=\langle\bar{z}, z\rangle+h(z, u)
$$

where $z \in \mathbb{C}^{n}, w=u+i v$ with $u, v \in \mathbb{R}^{d}$, and $\left\langle\bar{z}, z^{\prime}\right\rangle=\left({ }^{t} \bar{z} A_{1} z^{\prime}, \ldots,{ }^{t} \bar{z} A_{d} z^{\prime}\right)$. Assume the Hermitian matrices $A_{1}, \ldots, A_{d}$ satisfy conditions $(\mathfrak{a})$ and $(\mathfrak{b})$. The proof of Theorem 17 relies on the following step. 
4.1. The basic identity. Let $(F, G): \mathbb{C}^{n+d} \rightarrow \mathbb{C}^{n} \times \mathbb{C}^{d}$ be in $\operatorname{Aut}(M, 0)$. Since $(F, G)$ maps $M$ into $M$, we get

$$
\begin{aligned}
\operatorname{Im} G(z, u+i[\langle\bar{z}, z\rangle+h(z, u)]) \\
=\quad\langle\overline{F(z, u+i[\langle\bar{z}, z\rangle+h(z, u)])}, F(z, u+i[\langle\bar{z}, z\rangle+h(z, u)])\rangle \\
\quad+h(F(z, u+i[\langle\bar{z}, z\rangle+h(z, u)]), \operatorname{Re} G(z, u+i[\langle\bar{z}, z\rangle+h(z, u)]))
\end{aligned}
$$

We first follow [10]: this equality between two smooth functions leads to the equality of their Taylor expansions. Let us write $F(z, w)=a z+b w+\ldots$ and $G(z, w)=\alpha z+\beta w+\ldots$ where $a, b, \alpha, \beta$ are complex linear maps, and the dots represent higher order terms. Injecting in the previous equality, we identify the first coefficients:

- the right member contains no linear term, so $\alpha=0$ and $\operatorname{Im} \beta=0$;

- the Hermitian terms with respect to $z$ give $\beta\langle\bar{z}, z\rangle=\langle\overline{a z}, a z\rangle$.

Assigning weight one to $z$ and weight two to $w$, we decompose $F$ and $G$ into weighted homogeneous polynomials:

$$
F=\sum_{q=0}^{+\infty} F_{q} \quad, \quad G=\sum_{q=0}^{+\infty} G_{q}
$$

where $F_{q}\left(t z, t^{2} w\right)=t^{q} F_{q}(z, w)$ and the same for $G_{q}$. We thus know that

$$
\left\{\begin{array}{l}
F_{0}=0, F_{1}=a z \\
G_{0}=0, G_{1}=0, G_{2}=\beta w \text { where } \operatorname{Im} \beta=0 \text { and } \beta\langle\bar{z}, z\rangle=\langle\overline{a z}, a z\rangle
\end{array}\right.
$$

(notice that, since $(F, G)$ is biholomorphic at the origin, $a$ and $\beta$ must be invertible).

In the same way, for $q>2$, isolating the $q$-th component in the left member gives

$$
\operatorname{Im} G_{q}(z, u+i\langle\bar{z}, z\rangle)+\text { terms in } G_{r} \text { with } r<q
$$

and in the right member

$\left\langle\overline{F_{q-1}(z, u+i\langle\bar{z}, z\rangle)}, a z\right\rangle+\left\langle\overline{a z}, F_{q-1}(z, u+i\langle\bar{z}, z\rangle)\right\rangle+$ terms in $F_{r-1}$ with $r<q$

So we obtain that the sequences $\left(F_{q}\right)$ and $\left(G_{q}\right)$ satisfy the following recurring system:

$\forall q>2, \operatorname{Re}\left(i G_{q}+2\left\langle\overline{F_{q-1}}, a z\right\rangle\right)_{\mid v=\langle\bar{z}, z\rangle}=$ function of $G_{r}, F_{r-1}$ with $r<q$

Assume that $F_{0}, \ldots, F_{q-2}$ and $G_{0}, \ldots, G_{q-1}$ are known. Then the difference between two solutions $\left(F_{q-1}, G_{q}\right)$ and $\left(\tilde{F}_{q-1}, \tilde{G}_{q}\right)$ of (6) is a weighted homogeneous polynomial solution of the equation $\operatorname{Re}(i g+2\langle\bar{f}, a z\rangle)_{\mid v=\langle\bar{z}, z\rangle}=0$. We will prove that the polynomial solutions of this homogeneous equation 
are of weight $q$ bounded by some $q_{0}$. In particular, a solution of the recurring system (66) is uniquely determined by the initial values $F_{0}, \ldots, F_{q_{0}-1}$ and $G_{0}, \ldots, G_{q_{0}}$. Let $k$ be the maximal degree appearing in terms of weight $q_{0}$ : then if two elements of $\operatorname{Aut}(M, 0)$ have the same $k$-jet at the origin, they are equal.

Thus we aim to prove that (weighted homogeneous) polynomial solutions of the equation $\operatorname{Re}(i g+2\langle\bar{f}, a z\rangle)_{\mid v=\langle\bar{z}, z\rangle}=0$ are of bounded degree. Using that $a$ and $\beta$ are invertible linear maps and that $\beta$ is real satisfying $\beta\langle\bar{z}, z\rangle=$ $\langle\overline{a z}, a z\rangle$, we may replace $f$ by $a f$ and $g$ by $\beta g$ and get

$$
\operatorname{Re}(i g+2\langle\bar{f}, z\rangle)_{\mid v=\langle\bar{z}, z\rangle}=0
$$

Theorem 17 will follow from

Theorem 18. The polynomial solutions of (7) are of bounded degree. More precisely, the partial degree in the $z_{j}$ variables is bounded by 2.

4.2. Towards a system of PDE. Assume that $(f, g)$ is a holomorphic solution of (17). We decompose $f=\sum_{p=0}^{+\infty} f_{p}$ and $g=\sum_{p=0}^{+\infty} g_{p}$ in the following way:

$$
f_{p}(z, w)=\sum_{J} a_{J}^{(p)}(z) w^{J}, g_{p}(z, w)=\sum_{J} b_{J}^{(p)}(z) w^{J}
$$

where $a_{J}^{(p)}(z), b_{J}^{(p)}(z)$ are holomorphic homogeneous polynomials in $z$ of degree $p$. We may then rewrite (7) as

(77)

$$
\operatorname{Re}\left(i \sum_{p} g_{p}(z, u+i\langle\bar{z}, z\rangle)+2\left\langle\sum_{p} \overline{f_{p}(z, u+i\langle\bar{z}, z\rangle)}, z\right\rangle\right) \equiv 0
$$

In (17), we sort the terms by bidegree $(k, l)$ in $(z, \bar{z})$, that is, grouping the $z^{K} \bar{z}^{L}$ such that $|K|=k$ et $|L|=l$.

(a) terms of bidegree $(0,0): \operatorname{Re}\left(i \sum_{J} b_{J}^{(0)}(z) u^{J}+0\right)$ i.e. the $b_{J}^{(0)}$ are real and

$$
\operatorname{Im}\left(g_{0}(u)\right) \equiv 0
$$

(b) terms of bidegree $\left.(1,0):\left(i g_{1}(z, u)+2\left\langle\overline{f_{0}(u)}, z\right)\right\rangle\right)+0$ because the conjugate $\overline{\left(i g_{1}(z, u)+2\left\langle\overline{f_{0}(u)}, z\right\rangle\right)}$ does not contain such terms; so

$$
i g_{1}(z, u)+2\left\langle\overline{f_{0}(u)}, z\right\rangle \equiv 0
$$

(c) terms of bidegree $(k, 0)$ for $k \geq 2$ : $\operatorname{Re}\left(i g_{k}(z, u)\right)$; since $g_{k}$ is holomorphic, $\operatorname{Re}\left(i g_{k}(z, u)\right) \equiv 0$ implies $g_{k}=0$, thus

$$
\forall k \geq 2, g_{k}=0
$$


(d) terms of bidegree $(1,1)$ : they only involve $g_{0}$ and $f_{1}$. Note that

$$
\begin{aligned}
g_{0}(z, u+i\langle\bar{z}, z\rangle) & =\sum_{J} b_{J}^{(0)}(u+i\langle\bar{z}, z\rangle)^{J} \\
& =\sum_{J=\left(j_{1}, \ldots, j_{s}\right)} b_{J}^{(0)} \prod_{s=1}^{d}\left(u_{s}+i\langle\bar{z}, z\rangle_{s}\right)^{j_{s}}
\end{aligned}
$$

and the $(1,1)$-part is $\sum_{J=\left(j_{1}, \ldots, j_{s}\right)} b_{J}^{(0)} \sum_{l=1}^{d} j_{l} u_{l}^{j_{l}-1} i\langle\bar{z}, z\rangle_{l} \prod_{s \neq l} u_{s}^{j_{s}}$. We thus introduce the operator $\Delta$, associating to any differentiable map $\varphi(z, u)$ the derivative of $\varphi$ with respect to $u$ at point $(z, u)$ in the direction $\langle\bar{z}, z\rangle$, that is

$$
\Delta \varphi(z, u)=\partial_{u} \varphi_{(z, u)} \cdot\langle\bar{z}, z\rangle
$$

Note that $\Delta^{k} \varphi(z, u)=\partial_{u}^{k} \varphi_{(z, u)} \cdot(\langle\bar{z}, z\rangle, \ldots,\langle\bar{z}, z\rangle)$ when it is well defined. This gives

$$
\operatorname{Re}\left(-\Delta g_{0}(z, u)+2\left\langle\overline{f_{1}(z, u)}, z\right\rangle\right) \equiv 0
$$

(e) terms of bidegree $(2,1)$ : in $i \sum_{p} g_{p}(z, u+i\langle\bar{z}, z\rangle)+2\left\langle\sum_{p} \overline{f_{p}(z, u+i\langle z, z\rangle)}, z\right\rangle$, they only come from the $g_{1}$ and the $f_{0}$ parts; in the conjugate, only from the $f_{2}$ part. More precisely, we get

$$
-\Delta g_{1}(z, u)+2\left\langle\bar{z}, f_{2}(z, u)\right\rangle-2 i\left\langle\overline{\Delta f_{0}(z, u)}, z\right\rangle \equiv 0
$$

(f) terms of bidegree $(k+1,1)$ for $k \geq 2$ :

$$
-\Delta g_{k}(z, u)+2\left\langle\bar{z}, f_{k+1}(z, u)\right\rangle \equiv 0
$$

(g) terms of bidegree $(2,2)$ : now we are interested in the terms of bidegree $(2,2)$ in $(u+i\langle\bar{z}, z\rangle)^{J}$, and they are given by $\frac{(i)^{2}}{2 !} \Delta^{2}$. Consequently we obtain $\operatorname{Re}\left(-\frac{i}{2} \Delta^{2} g_{0}(z, u)+2\left\langle\overline{i \Delta f_{1}(z, u)}, z\right\rangle\right)$, and thus

$$
\operatorname{Im}\left(\Delta^{2} g_{0}(z, u)+4\left\langle\overline{\Delta f_{1}(z, u)}, z\right\rangle\right) \equiv 0
$$

(h) terms of bidegree $(3,2)$ :

$$
\left.-\frac{i}{2} \Delta^{2} g_{1}(z, u)+2 i\left\langle\bar{z}, \Delta f_{2}(z, u)\right\rangle-\left\langle\overline{\Delta^{2} f_{0}(z, u)}, z\right\rangle\right) \equiv 0
$$

(i) terms of bidegree $(3,3)$ : as previously, the terms of bidegree $(3,3)$ in $(u+i\langle\bar{z}, z\rangle)^{J}$ are given by applying $\frac{(i)^{3}}{3 !} \Delta^{3}$, thus

$$
\operatorname{Re}\left(\Delta^{3} g_{0}-6\left\langle\overline{\Delta^{2} f_{1}}, z\right\rangle\right) \equiv 0
$$

From (드) and (ㅌ) , we obtain for $k \geq 3:\left\langle\bar{z}, f_{k}(z, u)\right\rangle \equiv 0$, or equivalently (since $f_{k}$ is holomorphic in $z$ ) that for all $(z, u), \forall z^{\prime},\left\langle z^{\prime}, f_{k+1}(z, u)\right\rangle=0$. According to condition $(\mathfrak{b})$, we have $f_{k+1}(z, u)=0$ thus

$$
\forall k \geq 3, f_{k}=0
$$

and so the only possibly non-zero parts are $g_{0}, g_{1}, f_{0}, f_{1}, f_{2}$. 
- Eliminate $g_{1}$ in (因):

by (b),$g_{1}(z, u)=2 i\left\langle\overline{f_{0}(u)}, z\right\rangle$, so we have $\Delta g_{1}(z, u)=2 i\left\langle\overline{\Delta f_{0}(z, u)}, z\right\rangle$, and then (远) becomes

$$
\left\langle\bar{z}, f_{2}(z, u)\right\rangle \equiv 2 i\left\langle\overline{\Delta f_{0}(z, u)}, z\right\rangle
$$

- Eliminate $g_{1}$ in (h):

since $\Delta g_{1}(z, u)=2 i\left\langle\overline{\Delta f_{0}(z, u)}, z\right\rangle$, we obtain $\left\langle\bar{z}, \Delta f_{2}(z, u)\right\rangle \equiv 0$ and thus

$$
\left\langle\overline{\Delta^{2} f_{0}(z, u)}, z\right\rangle \equiv 0
$$

- Eliminate $\operatorname{Im} g_{0}$ in (g):

$\operatorname{Im} g_{0}(u)=0$ means the $b_{J}^{(0)}$ are real, thus $\operatorname{Im}\left(\Delta^{k} g_{0}(z, u)\right) \equiv 0$ for all $k$. Then (g) becomes

$$
\operatorname{Im}\left(\left\langle\overline{\Delta f_{1}(z, u)}, z\right\rangle\right) \equiv 0
$$

- Eliminate $\operatorname{Im} g_{0}$ in (1):

applying $\Delta^{2}$ to (did) gives $2 \operatorname{Re}\left(\Delta^{2}\left\langle\overline{f_{1}(z, u)}, z\right\rangle\right)=\operatorname{Re}\left(\Delta^{3} g_{0}(z, u)\right)$ and by replacing in (ii), we get

$$
\operatorname{Re}\left(\Delta^{3} g_{0}(z, u)\right) \equiv 0
$$

Finally, we obtain that $(f, g)$ is a polynomial solution of (7) if and only if the $f_{k}$ and $g_{k}$ form a polynomial solution of the following system:

$$
\left\{\begin{aligned}
&(8 a) \quad \forall k \geq 3, f_{k}=0 \text { and } g_{k-1}=0 \\
&(8 b)\left\{\begin{array}{l}
i g_{1}+2\left\langle\bar{f}_{0}, z\right\rangle=0 \\
\left\langle\bar{z}, f_{2}\right\rangle=2 i\left\langle\overline{\Delta f_{0}}, z\right\rangle \\
\left\langle\overline{\Delta^{2} f_{0}}, z\right\rangle=0
\end{array}\right. \\
&(8 c)\left\{\begin{array}{l}
\operatorname{Im} g_{0}=0 \\
2 \operatorname{Re}\left(\left\langle\bar{f}_{1}, z\right\rangle\right)-\operatorname{Re}\left(\Delta g_{0}\right)=0 \\
\operatorname{Im}\left\langle\overline{\Delta f_{1}}, z\right\rangle=0 \\
\operatorname{Re} \Delta^{3} g_{0}=0
\end{array}\right.
\end{aligned}\right.
$$

where all maps are evaluated on $\{\operatorname{Im} w=0\}$, that is at $(z, u)$.

Note that $(8 a)$ exactly means that the partial degree of $(f, g)$ in the $z_{j}$ variables is at most 2 .

4.3. The solution space of (8) is finite dimensional. The maps $f_{0}$ and $g_{0}$ do not depend on $z$. We decompose $f_{1}, f_{2}$ and $g_{1}$ in the following way:

$$
f_{1}(z, u)=\sum_{J} a^{(1)}(z)_{J} u^{J}=\sum_{J}\left(\sum_{i=1}^{n} \alpha_{J, i} z_{i}\right) u^{J}=\sum_{i=1}^{n}\left(\sum_{J} \alpha_{J, i} u^{J}\right) z_{i}
$$

thus we set $f_{1}(z, u)=\sum_{i=1}^{n} \phi_{i}(u) z_{i}$ and similarly $g_{1}(z, u)=\sum_{i=1}^{n} \psi_{i}(u) z_{i}$ and $f_{2}(z, u)=\sum_{i \leq j} \Phi_{i, j}(u) z_{i} z_{j}$. Note that $\Delta f_{1}(z, u)=\sum_{i=1}^{n} \Delta \phi_{i}(z, u) z_{i}$, and the same for $g_{1}$ and $f_{2}$. 
All the equalities in (8b)-(8s) are polynomials in $z$ and $\bar{z}$. If all their coefficients are equated to zero, then we get an overdeterminated homogeneous system of linear partial differential equations in the $n$ components of $\overline{f_{0}}$, $\operatorname{Re} \phi_{i}, \operatorname{Im} \phi_{i}, \Phi_{i, j}$ and the $d$ components of $\operatorname{Re} g_{0}, \operatorname{Im} g_{0}, \psi_{i}(i, j=1, \ldots, n)$ with constant coefficients:

$$
P(D) \Theta=0 \text { on } \Omega \subset \mathbb{R}^{d}
$$

where the $m$ components of $\Theta$ are precisely the unknown functions, $D=$ $\left(\frac{\partial}{\partial u_{1}}, \ldots, \frac{\partial}{\partial u_{d}}\right), P=\left(P_{r, s}\right)$ is a $l \times m$-rectangular matrix with polynomial entries $P_{r, s} \in \mathbb{C}\left[X_{1}, \ldots, X_{d}\right]$, and $\Omega$ is an open convex neighborhood of the origin.

As for linear ordinary differential equations with constant coefficients, we begin with looking at the exponential solutions. Set $\Theta(u)=e^{\zeta \cdot u} \cdot \theta$, where the vector $\theta$ is independent of $u$ and $\zeta \cdot u=\sum_{j=1}^{d} \zeta_{j} u_{j}$ : it is a non-trivial solution of (9) if and only if $P(\zeta) \cdot \theta=0$. Let $V_{P}=\left\{\zeta=\left(\zeta_{1}, \ldots, \zeta_{d}\right) \in\right.$ $\left.\mathbb{C}^{d} / \operatorname{rank}(P(\zeta))<m\right\}$ be the characteristic set of the system (9). Note that $\zeta \in V_{P}$ exactly means that $\zeta$ induces a complex valued non-trivial exponential solution of (9).

Lemma 19. The only possible characteristic value for the system (9) is 0 .

Proof. Note that if we set $g_{0}^{r}(u)=\operatorname{Re}\left(g_{0}\right)(u), g_{0}^{i}(u)=\operatorname{Im}\left(g_{0}\right)(u)$, $f_{1}^{r}(z, u)=\operatorname{Re}\left(f_{1}\right)(z, u)=\sum_{i=1}^{n}\left(\sum_{J} \operatorname{Re}\left(\alpha_{J, i}\right) u^{J}\right) z_{i}, f_{1}^{i}(z, u)=\operatorname{Im}\left(f_{1}\right)(z, u)$, then

$\underline{(8 \mathrm{~g})}) \Longleftrightarrow\left\{\begin{array}{l}g_{0}^{i}=0 \\ 2\left\langle f_{1}^{r}(\bar{z}, u)-i f_{1}^{i}(\bar{z}, u), z\right\rangle+2\left\langle\bar{z}, f_{1}^{r}(z, u)+i f_{1}^{i}(z, u)\right\rangle-\Delta\left(g_{0}^{r}\right)(z, u)=0 \\ \left\langle\Delta\left(f_{1}^{r}\right)(\bar{z}, u)-i \Delta\left(f_{1}^{i}\right)(\bar{z}, u), z\right\rangle-\left\langle\bar{z}, \Delta\left(f_{1}^{r}\right)(z, u)+i \Delta\left(f_{1}^{i}\right)(z, u)\right\rangle=0 \\ \Delta^{3} g_{0}^{r}=0\end{array}\right.$

Assume that $\Theta(u)=e^{\zeta \cdot u} \cdot \theta$ is solution of (9) with $\zeta \in \mathbb{C}^{d}$ and $\theta$ a complex vector. By definition of $\Theta$, this means we can write

$$
\begin{gathered}
\overline{f_{0}}(u)=e^{\zeta \cdot u} \cdot \tilde{f}_{0}, f_{2}(z, u)=e^{\zeta \cdot u} \cdot \tilde{f}_{2}(z), g_{1}(z, u)=e^{\zeta \cdot u} \cdot \tilde{g}_{1}(z) \\
g_{0}^{r}(u)=e^{\zeta \cdot u} \cdot \tilde{g}_{0}^{r}, g_{0}^{i}(u)=0, f_{1}^{r}(z, u)=e^{\zeta \cdot u} \cdot \tilde{f}_{1}^{r}(z), f_{1}^{i}(z, u)=e^{\zeta \cdot u} \cdot \tilde{f}_{1}^{i}(z)
\end{gathered}
$$

Assume $\zeta \neq 0$ and check that the non-degeneracy hypothesis implies $\tilde{f}_{0}=$ $\tilde{f}_{1}^{r}=\tilde{f}_{1}^{i}=\tilde{f}_{2}=0$ and $\tilde{g}_{0}^{r}=\tilde{g}_{0}^{i}=\tilde{g}_{1}=0$.

Since $\overline{\Delta f_{0}(u)}=\Delta \overline{f_{0}}(u)=(\zeta \cdot\langle\bar{z}, z\rangle) e^{\zeta \cdot u} \cdot \tilde{f}_{0}$, (8) b) becomes

$$
\left\{\begin{array}{l}
i \tilde{g}_{1}(z)+2\left\langle\tilde{f}_{0}, z\right\rangle=0 \\
\left\langle\bar{z}, \tilde{f}_{2}(z)=0\right. \\
(\zeta \cdot\langle\bar{z}, z\rangle)^{2}\left\langle\tilde{f}_{0}, z\right\rangle=0
\end{array}\right.
$$

Condition $(\mathfrak{a})$ gives $\zeta \cdot\langle\bar{z}, z\rangle$ is not identically zero since $\zeta \neq 0$, hence $\left\langle\tilde{f}_{0}, z\right\rangle=$ 0 and condition $(\mathfrak{b})$ implies $\tilde{f}_{0}=0$; thus $\tilde{g}_{1}=0$. Since $\tilde{f}_{2}$ is holomorphic, we also get by condition $(\mathfrak{b})$ that $\tilde{f}_{2}=0$. 
Similarly, for (85) we obtain $\tilde{g}_{0}^{r}=\tilde{g}_{0}^{i}=0$, and the two remaining equations become

$$
\left\langle\tilde{f}_{1}^{r}(\bar{z})-i \tilde{f}_{1}^{i}(\bar{z}), z\right\rangle \pm\left\langle\bar{z}, \tilde{f}_{1}^{r}(z)+i \tilde{f}_{1}^{i}(z)\right\rangle=0
$$

Since $\tilde{f}_{1}^{r}$ and $\tilde{f}_{1}^{i}$ are holomorphic, by condition $(\mathfrak{b})$ we also get $\tilde{f}_{1}^{r} \pm i \tilde{f}_{1}^{i}=0$.

We conclude by means of the following statement:

Theorem 20. 21] In the situation described above, the following assertions are equivalent:

a) $\mathbb{C}\left[X_{1}, \ldots, X_{d}\right]^{m} / P^{T} \cdot \mathbb{C}\left[X_{1}, \ldots, X_{d}\right]^{l}$ is finite-dimensional;

b) the solution space $S=\left\{y \in \mathcal{C}^{\infty}\left(\mathbb{R}^{d}, \mathbb{C}\right) \mid P(D) y=0\right\}$ is finite-dimensional;

c) the characteristic set $V_{P}$ is of dimension zero.

Remark 21. If these equivalent conditions are satisfied, then the solution space $S$ has a direct sum decomposition (integral representation):

$$
S=\bigoplus_{\zeta \in V_{P}} \mathcal{P}(\zeta) e^{\zeta \cdot u}
$$

where $\mathcal{P}(\zeta) \subset \mathbb{C}\left[X_{1}, \ldots, X_{d}\right]^{m}$ are finitely dimensional spaces of polynomials, depending on $P$. In the general situation, only assuming that $V_{P} \neq \mathbb{C}^{d}$, the Fundamental Principle of Ehrenpreis-Palamodov states that every smooth solution is still the limit of a sequence of exponential-polynomial solutions ([14], Theorem 7.6.14).

Thus the solution space of (8) is finite dimensional. In particular the degrees of the polynomial solutions are bounded by a single constant, depending only on the system, that is, on the Levi map of the submanifold at the origin.

4.4. Identifying the problem. Following the proof of Beloshapka, page 242 , the degree with respect to $u$ of the polynomial solution of (8 $\mathrm{b}), f_{0}$ (respectively $f_{2}$ ), is claimed to be at most 1 (respectively 0 ). One writes

$$
\begin{aligned}
& f_{0}=a_{0}+a_{1}+\ldots, \\
& f_{2}=A_{0}+A_{1}+\ldots,
\end{aligned}
$$

each of $a_{p}$ (respectively $A_{q}$ ) beeing of degree $p$ (respectively $q$ ) with respect to $u$. One writes

$$
a_{p}=\sum a_{j_{1}} \ldots j_{p} u_{j_{1}} \ldots u_{j_{p}},
$$

where the coefficients $a_{j_{1}} \cdots j_{p}$ are symmetric with respect to the indices.

$$
A_{q}=\sum A_{j_{1}} \ldots j_{q} u_{j_{1}} \ldots u_{j_{q}},
$$


where the coefficients $A_{j_{1}} \cdots j_{q}$ are symmetric with respect to the indices. Rewriting then (80) with the help of (10), (11), (12) and (13), one obtains

$$
\begin{gathered}
<\bar{z}, A_{j_{1} \cdots j_{p-1}}>=2 i p \sum_{q}<\bar{z}, z>_{q}<\overline{a_{j_{1} \cdots j_{p-1} q}}, z>. \\
p(p-1) \sum_{q}<\bar{z}, z>_{q_{1}}<\bar{z}, z>_{q_{2}}<\overline{a_{j_{1} \cdots j_{p-1} q_{1} q_{2}}}, z>=0 .
\end{gathered}
$$

Assume now that (14) and (15) have a nonzero solution for some $p>1$, that is, there exists an homogeneous solution of (8b). Then Beloshapka proposes to set, for $s>0$,

$$
A_{l_{1} \ldots l_{s} j_{1} \cdots j_{p-1}}=A_{j_{1}} \cdots j_{p-1}, \quad a_{l_{1} \ldots l_{s} j_{1}} \cdots j_{p}=a_{j_{1}} \cdots j_{p}
$$

Beloshapka then claims that the homogeneous polynomial obtained by (16) gives a nonzero homogeneous solution of (8/b) of arbitrarily degree, which would lead to a contradiction. The problem is that these coefficients $A_{l_{1} \ldots l_{s} j_{1}} \cdots j_{p-1}$ and $a_{l_{1} \ldots l_{s} j_{1}} \cdots j_{p}$ are NOT symmetric with respect to the indices.

Remark 22. To our knowledge, the bound $k=2(1+\operatorname{codim} M)$ obtained by Zaitvev in 23 . seems to be the best up to now for generic submanifolds that are real-analytic and nondegenerate in the sense of Beloshapka.

\section{REFERENCES}

[1] M.S. Baouendi, P. Ebenfelt, L.P. Rothschild: Real Submanifolds in Complex Space and their Mappings. Princeton University Press, 1999.

[2] M.S. Baoudendi, P. Ebenfelt, L.P. Rothschild, CR automorphisms of real analytic CR manifolds in complex space, Comm. Anal. Geom. 6 (1998), 291-315.

[3] V.K. Beloshapka: Finite dimensionality of the group of automorphisms of a realanalytic surface. Math. USSR Izvestiya 32(2) 239-242, 1989.

[4] V.K. Beloshapka: A uniqueness theorem for automorphisms of a nondegenerate surface in a complex space. (Russian) Mat. Zametki 47(3) 17-22, 1990; transl. Math. Notes 47(3) 239-243, 1990.

[5] F. Bertrand, L. Blanc-Centi, Stationary holomorphic discs and finite jet determination problems, Math. Ann. 358 (2014), 477-509.

[6] F. Bertrand, L. Blanc-Centi, F. Meylan, Stationary discs and finite jet determination for non-degenerate generic real submanifolds Adv. Math. 343 (2019), 910-934.

[7] L. Blanc-Centi, F. Meylan: Chern-Moser operators and weighted jet determination problems in higher codimension, preprint, arXiv:1712.00295.

[8] M.S. Baouendi, N. Mir, L.P. Rothschild, Reflection Ideals and mappings between generic submanifolds in complex space, J. Geom. Anal. 12 (2002), 543-580.

[9] A. Boggess: CR Manifolds and the Tangential Cauchy-Riemann Complex. CRC Press, 1991.

[10] S.S. Chern, J.K. Moser: Real hypersurfaces in complex manifolds, Acta Math. 133(34) 219-271 (1974).

[11] P. Ebenfelt, Finite jet determination of holomorphic mappings at the boundary, Asian J. Math. 5 (2001), 637-662. 
[12] P. Ebenfelt, B. Lamel, Finite jet determination of $C R$ embeddings, J. Geom. Anal. 14 (2004), 241-265.

[13] P. Ebenfelt, B. Lamel, D. Zaitsev, Finite jet determination of local analytic CR automorphisms and their parametrization by 2-jets in the finite type case, Geom. Funct. Anal. 13 (2003), 546-573.

[14] L. Hörmander: An Introduction to Complex Analysis in Several Variables, Third Edition. North Holland, 1990.

[15] R. Juhlin, Determination of formal CR mappings by a finite jet, Adv. Math. 222 (2009), 1611-1648.

[16] R. Juhlin, B. Lamel, Automorphism groups of minimal real-analytic CR manifolds, J. Eur. Math. Soc. (JEMS) 15 (2013), 509-537.

[17] S.-Y. Kim, D. Zaitsev, Equivalence and embedding problems for CR-structures of any codimension, Topology 44 (2005), 557-584.

[18] M. Koláŕ, F. Meylan, D. Zaitsev, Chern-Moser operators and polynomial models in CR geometry, Adv. Math. 263 (2014), 321-356.

[19] B. Lamel, N. Mir, Finite jet determination of CR mappings, Adv. Math. 216 (2007), 153-177.

[20] N. Mir, D. Zaitsev, Unique jet determination and extension of germs of CR maps into spheres, preprint.

[21] U. Oberst: Finite Dimensional Systems of Partial Differential or Difference Equations. Adv. in Appl. Math. 17 337-356, 1996.

[22] A. Tumanov: Extremal discs and the regularity of CR mappings in higher codimension. Amer. J. Math. 123 445-473, 2001.

[23] D. Zaitsev: Germs of local automorphisms of real analytic CR structures and analytic dependence on the $k$-jets. Math. Res. Lett. 4(6) 823-842, 1997. 